\title{
Effect of Oligomeric Proanthocyanidin on the Antioxidant Status and Lung Function of Patients with Chronic Obstructive Pulmonary Disease
}

\author{
MENG-CHUN LU ${ }^{1,2,3}$, MEI-DUE YANG ${ }^{2,4,5,6}$, PING-CHUN LI $I^{4,6}$, \\ HSIN-YUAN FANG ${ }^{4,6}$, HUI-YING HUANG ${ }^{3}$, YIN-CHING CHAN ${ }^{1}$ and DA-TIAN BAU ${ }^{5,7^{*}}$ \\ ${ }^{1}$ Department of Food and Nutrition, Providence University, Taichung, Taiwan, R.O.C.; \\ Departments of ${ }^{2}$ Clinical Nutrition and ${ }^{4}$ Surgery, and ${ }^{5}$ Terry Fox Cancer Research Laboratory, \\ Translational Medicine Research Center, China Medical University Hospital, Taichung, Taiwan, R.O.C.; \\ Departments of ${ }^{3}$ Nutrition and ${ }^{6}$ Medicine, China Medical University, Taichung, Taiwan, R.O.C.; \\ ${ }^{7}$ Department of Bioinformatics and Medical Engineering, Asia University, Taichung, Taiwan, R.O.C.
}

\begin{abstract}
Background/Aim: Evidence exists that oxidative stress and oxidative damage play a pivotal role in chronic obstructive pulmonary disease (COPD). Oligomeric proanthocyanidins (OPCs) extracted from grape seeds have been shown to exhibit antioxidant capabilities greater than those of vitamin $C$ and $E$. The objective of this study was to evaluate the effects of OPCs on antioxidant status and lung function in patients with COPD. Patients and Methods: Patients were supplemented with $150 \mathrm{mg} /$ day $O P C(n=13)$ orally or with a placebo $(n=14)$ for 8 weeks in a randomized double-blind clinical design. Changes in anthropometric values, lung function, oxidative state, and lipid profiles were assessed after OPC or placebo treatment for 8 weeks. Results: The results showed that OPC supplementation significantly reduced the concentration of malondialdehyde, superoxide dismutase, and total cholesterol (TC)/highdensity lipoprotein cholesterol $(H D L-C)$ ratio. The concentration of $H D L-C$ significantly increased in the OPCtreated group. The plasma triglyceride, TC and low-density lipoprotein cholesterol values and the activities of catalase and glutathione peroxidase also decreased, but did not
\end{abstract}

This article is freely accessible online.

*These Authors contributed equally to this study.

Correspondence to: Da-Tian Bau, Yin-Ching Chan and Mei-Due Yang, Terry Fox Cancer Research Laboratory, Translational Medicine Research Center, China Medical University Hospital, 2 Yuh-Der Road, Taichung, 404 Taiwan, R.O.C. Tel: +886 422053366 Ext. 5805, e-mail: datian@mail.cmuh.org.tw/artbau2@gmail.com

Key Words: Oxidative status, chronic obstructive pulmonary disease, lipid profiles, lung function, oligomeric proanthocyanidin. significantly differ between the OPC-and placebo-treated groups. Lung function was not significantly different between the two groups after 8 weeks. Conclusion: OPC supplementation was effective in increasing the antioxidant capacity, in addition to improving the lipid profiles in patients with COPD.

Chronic obstructive pulmonary disease (COPD) has gained considerable attention due to its consequential systemic manifestations and the comorbidities resulting therefrom (1). It is associated with the overproduction of reactive oxygen species (ROS) and reactive nitrogen species, leading to an imbalance of oxidants and antioxidants, thus causing oxidative stress (2). This may adversely affect patients with COPD who are already at an elevated risk of exposure to oxidants. Even if respiratory function tests are within normal limits, the oxidant burden in the lungs of patients with COPD is equivalent to that experienced by smokers (3). Lipid peroxidation (LPO) products, measured as thiobarbituric acid-reactive substances (TBARS), are found at higher levels in breath condensate and in the lungs of patients with stable COPD (4). In addition, such LPO products are negatively correlated with the marker of lung function, forced expiratory volume in 1 second (FEV1), suggesting that LPO plays a pivotal role in the decline of lung function (5). On the other hand, a significant correlation was found between a lower dietary intake of vitamin $\mathrm{C}$ and lower values of FEV1 (6). The Third National Health and Nutrition Examination Survey of the US population indicated that jointly considered, serum antioxidants vitamin $\mathrm{C}$, vitamin $\mathrm{E}$, selenium, and $\beta$-carotene correlate with lung function (6). The correlation between lung function and serum selenium is stronger among current smokers than former or non-smokers; in addition, among the smokers, their intake and serum level of $\beta$-carotene was less than those of the non-smokers, and the negative 
correlation was smoking dose-dependent (7). Therefore, oxidative stress may play a role in the health of patients with COPD. Various approaches have been developed for improving the determination of the antioxidant capacity of the lung tissues for patients with COPD and the clinical effectiveness of antioxidant compounds in the treatment of COPD has been discussed.

Grape seed extract contains biologically active polyphenolic flavonoids which have been demonstrated to be involved in many biological and pharmacological processes and serve as therapeutic agents against oxidative stress (8, 9). The beneficial effects of grape seeds emanate from their antioxidant activities, free radical-scavenging activities, and LPO inhibition $(10,11)$. Grape seed extract has cardioprotective effects, reducing oxidative stress and alleviating inflammation (12). In addition, extract from red grape seeds increases paraoxonase activity, mostly through increasing high-density lipoprotein cholesterol (HDL-C) and apolipoprotein A-I levels in patients with moderate hyperlipidemia (13). Oligomeric proanthocyanidins (OPCs) extracted from grape seeds have been shown to exhibit antioxidant capabilities greater than those of vitamin $\mathrm{C}$ and E (14). OPC usage might contribute to the improvement of red blood cell function in type 2 diabetes (15). Grape seed proanthocyanidin extract treatment was found to inhibit oxidative damage and apoptosis and to exert beneficial effects in reducing the incidence of steroid-induced osteonecrosis in rabbit models (16). OPCs may selectively protect against oxidative stress, maintain genomic integrity, and prevent programmed cell death in vivo (17). OPCs exhibit potentially beneficial effects in preventing oxidative stress in human health (18).

In a lung function study, Mao et al. reported that grape seed proanthocyanidin mediated antineoplastic mechanisms involving modulations of oncomirs and the efficacy of leucoselect phytosome against lung cancer (19). Hypoxic pulmonary hypertension is a serious disease that causes significant disability and a reduced life expectancy. Sustained hypoxia caused by primary lung diseases, including COPD, cystic fibrosis, diffuse interstitial fibrosis, bronchopulmonary dysplasia, radiation fibrosis, infiltrative lung tumors, and collagen vascular disease, leads to the development of increased pulmonary vascular resistance and pulmonary hypertension (19). Supplementation with grape seed procyanidin extract prevented the changes in hemodynamics and pulmonary vascular remodeling that occurred in rats exposed to hypoxia. Furthermore, the data revealed that the extract was able to attenuate consequent oxidative stress and the proliferation of pulmonary artery smooth muscle cells (20).

Patients with COPD tend to have higher levels of DNA and lipid oxidation, advanced protein oxidation products, and higher glutathione peroxidase (GSH-Px) activity (21). A substantial number of investigations have demonstrated a broad spectrum of pharmacological and therapeutic benefits of grape seed proanthocyanidins against oxidative stress and degenerative diseases such as cardiovascular dysfunction, acute and chronic stress, gastrointestinal distress, neurological disorders, and pancreatitis, as well as various stages of neoplastic processes and carcinogenesis including detoxification of carcinogenic metabolites (17). Yet the effects of OPCs on COPD cases are not well known. Consequently, this study evaluated the effects of OPCs on oxidative status and lung function in patients with COPD.

\section{Patients and Methods}

Patients and experimental design. Thirty-two outpatients with stable COPD from Tungs' Taichung Metro Harbor Hospital, Taichung, Taiwan, were invited to participate in this study. The patient selection criteria in this study were as follows: absence of kidney disease, liver disease, or infectious illnesses; and not having received antioxidant supplements; $N$-acetylcysteine; antibiotics such as tetracycline; or conventional medications such as bronchodilators, steroids, or anticough or antiphlegm agents in the previous month.

In this study, a double-blind design was applied, and patients were randomly divided into a placebo group $(n=14)$ and an experimental group $(n=13)$. Patients were randomly assigned to supplementation with either $150 \mathrm{mg} /$ day OPC (Sun Valley Research Inc., Canada, Sunshine Valley International Co., Ltd, Taiwan, ROC) or placebo for 8 weeks. The daily dose was administered as tablets packed in paper bags that patients were required to return for compliance assessment. The appearance of the supplementation for both groups was identical. Patients consumed supplements in three split dosages with a meal. They were instructed to maintain their oral medication, habitual eating, drinking, and lifestyle behavior. The purposes and procedures of this study were explained to the patients, and their written consent was obtained. The Human Research Ethics Committee at Providence University, Taichung, Taiwan, approved the study protocol (20030710-H01).

Characteristics and nutritional assessments. A trained dietitian assessed the characteristics of the patients, including age, sex, body composition and nutritional measurements. Anthropometric data of height, body weight, body fat, waist circumference, hip circumference, triceps skinfold thickness, arm circumference, arm muscle circumference, arm muscle area, upper arm fat area, and blood pressure were measured. Oxidative status, biochemical values and lung function, as well as dietary intake, were also examined. Sample collection and measurement were conducted at the baseline (week 0) and week 8. Fasting venous blood of the patients was withdrawn at baseline and week 8 by medical assistants. One part of each blood sample was centrifuged at $1200 \times g$ for $10 \mathrm{~min}$ (Hitachi 7170; Hitachi Instruments Ltd., Tokyo, Japan) to obtain serum samples for analyzing the levels of total cholesterol (TC), triglyceride (TG), HDL-C, and low-density lipoprotein cholesterol (LDL-C). TC, TG, HDL-C and LDL-C were analyzed using an automatic analyzer (Synchron CX-7 systems; Beckman, Porterville, CA, USA). Another part of the blood sample was immediately placed in a heparinized tube and centrifuged $1200 \times g$ at $4^{\circ} \mathrm{C}$ for 10 min to obtain plasma and erythrocyte for determining the oxidative status of the patients. 
Lung function and oxidative status analyses. Lung function tests were performed using a SENSORMEDICS Vmax22 computerized lung function measurement and analysis instrument (SENSORMEDICS, San Diego, CA, USA) to determine vital capacity, lung volume, and lung permeability in three parts: flow, volume, and loop; standard Ventolin or Proventil test bronchial provocation tests; lung volume with functional residual capacity and other testing methods as well as various pulmonary functions.

The oxidative status of the patients, including TBARS in the plasma and the activities of superoxidase dismutase (SOD), catalase, and glutathione peroxidase (GSH-Px) in the erythrocyte sample were measured as previously described (22). In the Tables, the data for SOD, catalase and GSH-Px activities are expressed as units/g protein.

Statistical analysis. The data are shown as the mean \pm standard error of mean (SEM). The significance of the differences between the OPC- and placebo-treated groups was examined by the MannWhitney test, while the Wilcoxon signed-rank test was employed to compare the difference between the data at the baseline and posttest. Moreover, $p$-values of less than 0.05 were considered statistically significant. These statistical calculations were performed using SPSS software (SPSS Inc., Chicago, IL, USA).

\section{Results}

Table I presents the characteristics of the patients. There was no significant difference in age or COPD morbidity years between the two groups. These patients were generally illiterate or primary school educated, and most of them were retired or described their occupation as relating to agriculture. Anthropometric measurements of height, weight, body mass index, body fat, waist circumference, hip circumference, triceps skinfold thickness, arm circumference, arm muscle circumference, arm muscle area, and upper arm fat area between the groups indicated no significant difference before or after the treatment; moreover, no difference in blood pressure was observed (data not shown). Neither group demonstrated significant differences in total calorie, protein, fat, carbohydrates, dietary fiber, cholesterol, or vitamin and mineral intake between baseline and week 8 (data not shown). FEV1, forced vital capacity (FVC), the FEV1/FVC ratio, and the peak expiratory flow rate for pulmonary function testing of the four lung functions revealed no significant intergroup differences in respiratory status (Table II). It was speculated that a short period of OPC supplementation might not yield a significant improvement in lung function in patients with COPD.

Table III presents the results of OPC supplementation for 8 weeks in terms of the oxidative states in patients with COPD. OPC supplementation reduced the concentration of MDA, a product of plasma lipid oxidation $(p<0.05)$. The MDA concentration in the OPC group $(5.87 \pm 0.27 \mathrm{nmol} / \mathrm{ml})$ was significantly lower than that in the control group $(7.95 \pm 0.41$ $\mathrm{nmol} / \mathrm{ml}$ ). The SOD activity measured at 8 weeks was significantly reduced in the OPC-treated group $(2.7 \pm 0.45$ $\mathrm{nmol} / \mathrm{ml})$ relative to the placebo-treated group $(5.37 \pm 0.87$
Table I. Characteristics of patients. There were no significant differences between the placebo- and oligomeric proanthocyanidin $(O P C)$-treated groups.

\begin{tabular}{lcc}
\hline Characteristic & Placebo & OPC \\
\hline Total patients & & \\
$\mathrm{n}$ & 14 & 13 \\
Age, years & & \\
$\quad$ Mean \pm SEM & $72 \pm 2$ & $71 \pm 2$ \\
$<60$ & $2(14.3)$ & $0(0.0)$ \\
$60-69$ & $3(21.4)$ & $5(38.5)$ \\
$70-80$ & $7(50.0)$ & $7(53.8)$ \\
$>80$ & $2(14.3)$ & $1(7.7)$ \\
COPD duration, years & & \\
$\quad$ Mean \pm SEM & $9.7 \pm 2.5$ & $11.1 \pm 2.3$ \\
Level of education, n $(\%)$ & & \\
Illiterate & $7(50.0)$ & $8(61.5)$ \\
Primary school & $7(50.0)$ & $3(23.1)$ \\
Secondary school & $0(0.0)$ & $1(7.7)$ \\
Higher school & $0(0.0)$ & $1(7.7)$ \\
Training school & $0(0.0)$ & $0(0.0)$ \\
Work status, $\mathrm{n}(\%)$ & & \\
Retired & $12(85.7)$ & $12(92.3)$ \\
At work & $2(14.3)$ & $1(7.7)$ \\
Current or past occupation, n (\%) & & \\
Public servant & $3(21.4)$ & $2(15.4)$ \\
Business & $1(7.1)$ & $1(7.7)$ \\
Industry & $3(21.1)$ & $3(23.1)$ \\
Agriculture & $5(35.7)$ & $7(53.8)$ \\
Service & $2(14.3)$ & $0(0.0)$ \\
\hline
\end{tabular}

COPD: Chronic obstructive pulmonary disease; SEM: standard error of mean.

$\mathrm{nmol} / \mathrm{ml})(p<0.05)$. Furthermore, the MDA level and SOD activity after OPC supplementation were significantly reduced compared with baseline. However, catalase and GSH-Px demonstrated no significant difference between the groups.

The plasma lipid levels of the patients are shown in Table IV. The OPC group displayed significantly elevated HDL-C levels $(p<0.05)$. The TC/HDL-C ratio in the OPC-treated group $(3.3 \pm 0.3)$ was significantly lower than that in the placebotreated group $(p<0.05)$, and was also statistically significantly lower than that at baseline. The levels of TC, TG, and LDL-C did not significantly differ between baseline and 8 weeks in the placebo-treated groups. The above results revealed that OPC supplemented at $150 \mathrm{mg} /$ day for 8 weeks improved the levels of antioxidant enzymes (MDA, SOD) and serum lipid profiles (HDL-C, TC/HDL-C ratio) of patients with COPD.

\section{Discussion}

Patients with COPD are often exposed to exogenous forms of free radicals from environmental pollutants and cigarette smoke. Hartmann et al. indicated a significant increase in systemic oxidative stress marker MDA, 8-hydroxy-2'- 
deoxyguanosine, advanced oxidation protein products, and antioxidant enzyme activity in patients with COPD, compared with healthy cohorts (21). In another study, MDA, a product of LPO, was found to be significantly higher in the exhaled breath condensate of 73 patients with COPD compared with healthy controls, patients with asthma, and patients with bronchiectasis; the same study also showed that MDA levels in COPD correlated inversely with $\mathrm{FEV}_{1}$ (23), suggesting a link with disease severity. Furthermore, increased LPO, as evidenced by increased MDA, and reduced catalase or SOD in patients with COPD were reported (24). Li et al. showed the levels of serum SOD and total antioxidant capacity in COPD patients with pulmonary hypertension were reduced when compared with those in patients without pulmonary hypertension, and were positively correlated with the 3-year survival rate (25).

Our study showed that OPC supplementation significantly reduced the concentration of MDA and the activity of SOD in patients with COPD, and this was possibly related to the antioxidant effects of grape seed polyphenols. Grape seed components possess not only anticoagulant but also antioxidative properties and may be useful in creating new therapeutic agents or dietary supplements. They may be helpful in both preventing and treating thromboembolic complications associated with multiple failures of hemostasis because the available therapeutic agents do not offer such dual effects (26). A series of studies was performed using grape seed extract to elucidate its cardioprotective ability in animals and humans. Grape seed extract supplementation improved cardiac functional assessment, including post-ischemic left ventricular function, reduced the myocardial infarct size, reduced ventricular fibrillation and ventricular tachycardia, reduced the amount of ROS, and reduced MDA formation in the heart perfusion (26). Du et al. confirmed that several antioxidant enzymes in cultured cardiomyocytes were induced by low micromolar concentrations of two major polyphenols isolated from grape seed extract (27). This chemically mediated up-regulation of cellular defense was accompanied by a markedly increased resistance to cardiac cell apoptosis elicited by ROS. As mentioned earlier, the efficient detoxification of ROS requires the coordinated action of various cellular antioxidant enzymes. Accordingly, the simultaneous induction of a range of key cellular antioxidant enzymes by grape seed polyphenols in cardiovascular cells may be an important mechanism underlying the protective effects of grape seed extract observed in various cardiovascular disorders (27). In this study, the SOD activity of patients with COPD was significantly reduced, and OPC may help such patients to return to a normal state of antioxidant-oxidant balance via enhancing the SOD capturing of ROS, and suppressing oxidative stress.

Grape seed polyphenols mainly contain proanthocyanidins. Generally, di- and trimeric proanthocyanidins, referred to as
Table II. Effect of oligomeric proanthocyanidin (OPC) supplementation for 8 weeks on lung function in patients with chronic obstructive pulmonary disease. Data are expressed as the mean \pm standard error of mean. There were no significant differences between the placebo and OPC groups.

\begin{tabular}{lccc}
\hline Measure & Week & Placebo $(\mathrm{n}=14)$ & OPC $(\mathrm{n}=13)$ \\
\hline $\mathrm{FEV}_{1}$ (\% pred) & 0 & $67.50 \pm 4.92$ & $65.92 \pm 6.11$ \\
& 8 & $69.71 \pm 6.38$ & $65.85 \pm 7.97$ \\
FVC (\% pred) & 0 & $44.00 \pm 4.10$ & $44.85 \pm 5.44$ \\
& 8 & $46.29 \pm 4.56$ & $43.92 \pm 5.39$ \\
FEV $_{1} /$ FVC (\%) & 0 & $51.50 \pm 4.41$ & $52.85 \pm 3.91$ \\
& 8 & $52.79 \pm 4.43$ & $53.54 \pm 4.79$ \\
PEFR (\% pred) & 0 & $45.29 \pm 3.47$ & $45.46 \pm 4.95$ \\
& 8 & $45.93 \pm 4.12$ & $45.54 \pm 4.49$ \\
\hline
\end{tabular}

$\mathrm{FEV}_{1}$ : Forced expiratory volume in 1 second; FVC: forced vital capacity; PEFR: peak expiratory flow rate; $\%$ pred: $\%$ predicted lung function.

Table III. Effect of oligomeric proanthocyanidin (OPC) supplementation for 8 weeks on oxidative state in patients with chronic obstructive pulmonary disease. Data are expressed as the mean \pm standard error of mean

\begin{tabular}{lccc}
\hline Measure & Week & Placebo $(\mathrm{n}=14)$ & OPC $(\mathrm{n}=13)$ \\
\hline MDA (nmol/ml) & 0 & $7.75 \pm 0.57$ & $7.12 \pm 0.37$ \\
& 8 & $7.95 \pm 0.41$ & $5.87 \pm 0.27 * \#$ \\
SOD (U/g protein) & 0 & $6.84 \pm 1.05$ & $6.19 \pm 0.85$ \\
& 8 & $5.37 \pm 0.87$ & $2.70 \pm 0.45^{* \#}$ \\
Catalase (U/g protein) & 0 & $15.29 \pm 1.01$ & $16.85 \pm 2.32$ \\
& 8 & $16.51 \pm 1.65$ & $13.42 \pm 1.25$ \\
GSH-Px (U/g protein) & 0 & $0.17 \pm 0.02$ & $0.17 \pm 0.01$ \\
& 8 & $0.19 \pm 0.03$ & $0.14 \pm 0.01$ \\
\hline
\end{tabular}

MDA: Malondialdehyde; SOD: superoxide dismutase; GSH-Px: glutathione peroxidase. Significantly different at $p<0.05$ compared to *placebo group and "baseline in the same group.

Table IV. Effect of oligomeric proanthocyanidin (OPC) supplementation for 8 weeks on plasma lipids in patients with chronic obstructive pulmonary disease. Data are expressed as the mean \pm standard error of mean.

\begin{tabular}{lccc}
\hline Measure & Week & Placebo $(\mathrm{n}=14)$ & OPC $(\mathrm{n}=13)$ \\
\hline Total cholesterol (mg/dl) & 0 & $205 \pm 10$ & $203 \pm 14$ \\
& 8 & $202 \pm 9$ & $198 \pm 13$ \\
Triglycerides (mg/dl) & 0 & $111 \pm 16$ & $116 \pm 15$ \\
& 8 & $125 \pm 21$ & $114 \pm 20$ \\
HDL-C (mg/dl) & 0 & $53 \pm 4$ & $58 \pm 4$ \\
& 8 & $47 \pm 4$ & $59 \pm 4^{*}$ \\
LDL-C (mg/dl) & 0 & $141 \pm 8$ & $136 \pm 13$ \\
& 8 & $126 \pm 7^{\#}$ & $120 \pm 12^{\#}$ \\
TC/HDL-C ratio & 0 & $4.1 \pm 0.3$ & $3.6 \pm 0.3$ \\
& 8 & $4.6 \pm 0.3 \#$ & $3.3 \pm 0.3^{* \#}$ \\
\hline
\end{tabular}

HDL: High-density lipoprotein cholesterol; LDL: low-density lipoprotein cholesterol; TC: total cholesterol. Significantly different at $p<0.05$ compared with *placebo group and ${ }^{\#}$ baseline in the same group. 
extractable proanthocyanidins or bioflavonoids, have proven to be highly bioavailable chemicals that provide excellent health benefits (28). In 2011, using an animal model, Zhang et al. demonstrated that deep vein thrombosis was significantly inhibited by grape seed proanthocyanidins (29). According to Chedea et al., grape seed extract may exhibit antioxidant or pro-oxidant activity, depending on the dose, administration duration, and other dietary components (30). Weseler et al. described various aspects of pleiotropic action on vascular health in humans through weekly supplementation with $200 \mathrm{mg} /$ day monomeric and oligomeric flavanols. In the monomeric and oligomeric flavanols group, serum TC and LDL levels decreased considerably; moreover, anti-inflammatory effects in blood toward ex vivo acquired bacterial endotoxin and a significant reduction of inflammatory gene expression in leukocytes were noted (31). Our results indicate that OPCs increased the serum HDL-C level and lowered the TC/HDL-C ratio of patients with COPD.

In conclusion, OPCs may improve the antioxidant status via increasing SOD and MDA action, and improving the serum lipid profiles of patients with COPD, but the effects on lung function should be further investigated in long-term studies.

\section{Conflicts of Interest}

The Authors declare no financial or non-financial conflicts of interest in regard to this study.

\section{Acknowledgements}

This study was partly supported by grants from the Ministry of Science and Technology, Taiwan (MOST 104-2320-B-126-005MY3), Ministry of Health and Welfare, Taiwan (MOHW107-TDUB-212-123004) and Taichung Veterans General Hospital and Providence University, Taichung, Taiwan (TCVGH-PU1038102). The Authors also greatly appreciate the technical assistance provided by Ms. Ying-Min Liu.

\section{References}

1 Barnes PJ and Celli BR: Systemic manifestations and comorbidities of COPD. Eur Respir J 33: 1165-1185, 2009.

2 Rahman I: Oxidative stress in pathogenesis of chronic obstructive pulmonary disease: cellular and molecular mechanisms. Cell Biochem Biophys 43: 167-188, 2005.

3 Inonu $\mathrm{H}$, Doruk S, Sahin S, Erkorkmaz U, Celik D, Celikel S and Seyfikli Z: Oxidative stress levels in exhaled breath condensate associated with COPD and smoking. Respir Care 57: 413-419, 2012.

4 Nowak D, Kasielski M, Antczak A, Pietras T and Bialasiewicz $\mathrm{P}$ : Increased content of thiobarbituric acid-reactive substances and hydrogen peroxide in the expired breath condensate of patients with stable chronic obstructive pulmonary disease: no significant effect of cigarette smoking. Respir Med 93: 389$396,1999$.
5 Tsukagoshi H, Shimizu Y, Iwamae S, Hisada T, Ishizuka T, Iizuka K, Dobashi K and Mori M: Evidence of oxidative stress in asthma and COPD: potential inhibitory effect of theophylline. Respir Med 94: 584-588, 2000.

6 Schwartz J and Weiss ST: Relationship between dietary vitamin $\mathrm{C}$ intake and pulmonary function in the First National Health and Nutrition Examination Survey (NHANES I). Am J Clin Nutr 59: 110-114, 1994.

$7 \mathrm{Hu} \mathrm{G}$ and Cassano PA: Antioxidant nutrients and pulmonary function: the Third National Health and Nutrition Examination Survey (NHANES III). Am J Epidemiol 151: 975-981, 2000.

8 Bagchi D, Garg A, Krohn RL, Bagchi M, Tran MX and Stohs SJ: Oxygen free radical scavenging abilities of vitamins $\mathrm{C}$ and $\mathrm{E}$, and a grape seed proanthocyanidin extract in vitro. Res Commun Mol Pathol Pharmacol 95: 179-189, 1997.

9 Sato M, Maulik G, Ray PS, Bagchi D and Das DK: Cardioprotective effects of grape seed proanthocyanidin against ischemic reperfusion injury. J Mol Cell Cardiol 31: 1289-1297, 1999.

10 Mackness MI, Mackness B, Durrington PN, Connelly PW and Hegele RA: Paraoxonase: biochemistry, genetics and relationship to plasma lipoproteins. Curr Opin Lipidol 7: 69-76, 1996.

11 Durrington PN, Mackness B and Mackness MI: Paraoxonase and atherosclerosis. Arterioscler Thromb Vasc Biol 21: 473-480, 2001.

12 Yousef MI, Saad AA and El-Shennawy LK: Protective effect of grape seed proanthocyanidin extract against oxidative stress induced by cisplatin in rats. Food Chem Toxicol 47: 1176-1183, 2009.

13 Argani H, Ghorbanihaghjo A, Vatankhahan H, Rashtchizadeh N, Raeisi S and Ilghami H: The effect of red grape seed extract on serum paraoxonase activity in patients with mild to moderate hyperlipidemia. Sao Paulo Med J 134: 234-239, 2016.

14 Uchiyama H, Uehara K, Nagashima T, Nakata A, Sato K, Mihara Y, Komatsu KI, Takanari J, Shimizu S and Wakame K: Global Liver gene expression analysis on a murine metabolic syndrome model treated by low-molecular-weight lychee fruit polyphenol [Oligonol(R)]. Anticancer Res 36: 3705-3713, 2016.

15 Visser J, van Staden PJ, Soma P, Buys AV and Pretorius E: The stabilizing effect of an oligomeric proanthocyanidin on red blood cell membrane structure of poorly controlled Type II diabetes. Nutr Diabetes 7: e275, 2017.

16 Song Q, Shi Z, Bi W, Liu R, Zhang C, Wang K and Dang X: Beneficial effect of grape seed proanthocyanidin extract in rabbits with steroid-induced osteonecrosis via protecting against oxidative stress and apoptosis. J Orthop Sci 20: 196-204, 2015.

17 Bagchi D, Swaroop A, Preuss HG and Bagchi M: Free radical scavenging, antioxidant and cancer chemoprevention by grape seed proanthocyanidin: an overview. Mutat Res 768: 69-73, 2014.

18 Ochs-Balcom HM, Grant BJ, Muti P, Sempos CT, Freudenheim JL, Browne RW, McCann SE, Trevisan M, Cassano PA, Iacoviello L and Schunemann HJ: Antioxidants, oxidative stress, and pulmonary function in individuals diagnosed with asthma or COPD. Eur J Clin Nutr 60: 991-999, 2006.

19 Mao JT, Xue B, Smoake J, Lu QY, Park H, Henning SM, Burns W, Bernabei A, Elashoff D, Serio KJ and Massie L: MicroRNA19a/b mediates grape seed procyanidin extract-induced antineoplastic effects against lung cancer. J Nutr Biochem 34: 118$125,2016$. 
20 Jin H, Liu M, Zhang X, Pan J, Han J, Wang Y, Lei H, Ding Y and Yuan Y: Grape seed procyanidin extract attenuates hypoxic pulmonary hypertension by inhibiting oxidative stress and pulmonary arterial smooth muscle cells proliferation. J Nutr Biochem 36: 81-88, 2016.

21 Hartmann SE, Pialoux V, Leigh R and Poulin MJ: Decreased cerebrovascular response to $\mathrm{CO} 2$ in post-menopausal females with COPD: role of oxidative stress. Eur Respir J 40: 13541361, 2012.

22 Sheu WH, Lee IT, Chen W and Chan YC: Effects of xylooligosaccharides in type 2 diabetes mellitus. J Nutr Sci Vitaminol (Tokyo) 54: 396-401, 2008.

23 Bartoli ML, Novelli F, Costa F, Malagrino L, Melosini L, Bacci E, Cianchetti S, Dente FL, Di Franco A, Vagaggini B and Paggiaro PL: Malondialdehyde in exhaled breath condensate as a marker of oxidative stress in different pulmonary diseases. Mediators Inflamm 2011: 891752, 2011.

24 Tavilani H, Nadi E, Karimi J and Goodarzi MT: Oxidative stress in COPD patients, smokers, and non-smokers. Respir Care 57: 2090-2094, 2012.

$25 \mathrm{Li} \mathrm{C}$, Yan L and Xu J: Correlations between lipid ratio/oxidative stress status in COPD patients and pulmonary hypertension as well as prognosis. Zhong Nan Da Xue Xue Bao Yi Xue Ban 41: 1168-1174, 2016.

26 Bijak M, Kolodziejczyk-Czepas J, Ponczek MB, Saluk J and Nowak P: Protective effects of grape seed extract against oxidative and nitrative damage of plasma proteins. Int J Biol Macromol 51: 183-187, 2012.
$27 \mathrm{Du}$ Y, Guo H and Lou H: Grape seed polyphenols protect cardiac cells from apoptosis via induction of endogenous antioxidant enzymes. J Agric Food Chem 55: 1695-1701, 2007.

28 Bagchi D, Bagchi M, Stohs SJ, Das DK, Ray SD, Kuszynski CA, Joshi SS and Pruess HG: Free radicals and grape seed proanthocyanidin extract: importance in human health and disease prevention. Toxicology 148: 187-197, 2000.

29 Zhang Y, Shi H, Wang W, Ke Z, Xu P, Zhong Z, Li X and Wang $S$ : Antithrombotic effect of grape seed proanthocyanidins extract in a rat model of deep vein thrombosis. J Vasc Surg 53: 743-753, 2011.

30 Chedea VS, Braicu C and Socaciu C: Antioxidant/prooxidant activity of a polyphenolic grape seed extract. Food Chem 121: 132-139, 2010.

31 Weseler AR, Ruijters EJ, Drittij-Reijnders MJ, Reesink KD, Haenen GR and Bast A: Pleiotropic benefit of monomeric and oligomeric flavanols on vascular health-a randomized controlled clinical pilot study. PLoS One 6: e28460, 2011.

Received March 15, 2018

Revised May 3, 2018

Accepted May 9, 2018 